\title{
A HETEROGENEIDADE DO GLIOBLASTOMA E TRATAMENTOS
}

\section{THE HETEROGENEITY OF GLIOBLASTOMA AND TREATMENTS}

\author{
Gabriela Corrêa de Sousa ${ }^{1}$ \\ Dorival Scantamburlo Junior ${ }^{2}$ \\ Luciana Estevam Simonato ${ }^{3}$
}

RESUMO: Objetivo: $O$ presente trabalho teve como objetivo descrever o glioblastoma, mostrando sua epidemiologia e características histológicas. Além disso, mostrar os tipos de exames diagnósticos e tratamentos disponíveis. Métodos: Foram realizados levantamentos de produções cientificas que abordassem o glioblastoma como tema central, escritos em inglês ou português, publicados de 2000 a 2020 . Conclusão: $O$ glioblastoma é um tumor cerebral primário muito prevalente em adultos e muitas vezes assintomático no início com sintomas inespecíficos tendo um diagnóstico difícil e tardio, com tratamento complexo. No entanto, o prognóstico da doença é ruim, apresenta uma sobrevida de 4 a 6 meses em pacientes que optam pelo não tratamento e 14 meses em média em pacientes com tratamento multimodal.

Palavras-chave: Glioblastoma. Heterogeneidade. Tratamentos.

ABSTRACT: Objective: This study aimed to describe glioblastoma, showing its epidemiology and histological characteristics. Also, show the types of diagnostic tests and treatments available. Methods: Surveys of scientific productions that addressed glioblastoma as a central theme, written in English or Portuguese, published from 2000 to 2020, were carried out. Conclusion: Glioblastoma is a primary brain tumor very prevalent in adults and often asymptomatic at first with nonspecific symptoms having a difficult and late diagnosis, with complex treatment. However, the prognosis of the disease is poor, with a survival of 4 to 6 months in patients who opt for no treatment and 14 months on average in patients with multimodal treatment.

Keywords: Glioblastoma. Heterogeneity. Treatments.

\section{INTRODUÇÃO}

O glioblastoma (GBM) é chamado o tumor cerebral primário maligno mais prevalente na população adulta, além disso, apresenta significativa agressividade e também comportamento invasivo. Sua classificação é de ordem primária, ou seja, a GBM que surge sem um precursor clínico conhecido e secundário, oriundo da

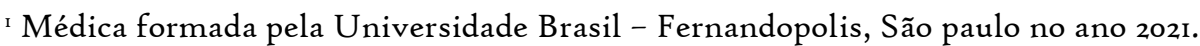
${ }^{2}$ Médico formado pela Universidade Brasil - Fernandopolis, São Paulo No ano 2021.

${ }^{3}$ Cirurgiã-dentista Doutorada em engenharia biomedica. Docente dos cursos de medicina e odontologia da Universidade Brasil- Fernandopolis, São Paulo. Mestre em Estomatologia. 
progressão molecular associada à elevação do grau de malignidade de um glioma prévio (BATISTA NETTO; SILVA e NASCIMENTO, 2019).

Sua incidência é entre 2 a 3 casos para 100.000 indivíduos, correspondentes a, aproximadamente, $14,9 \%$ dos tumores cerebrais primários e a $51 \%$ dos gliomas o que corresponde a $2 \%$ de todos os outros tipos de cânceres. Importa ainda dizer que a incidência quando é relacionada à idade, aumenta após os 40 anos apresenta uma média de 64 anos ao diagnóstico e um pico entre 75 e 80 anos, com 15,3 casos para ıо.оoо pessoas (BATISTA NETTO; SILVA e NASCIMENTO, 2019).

Estudo realizado por Aaron et al., (2020) aponta que os glioblastomas, junto com outros gliomas, surjam de células progenitoras neurogliais. A revisão de 2016 da classificação de tumores de SNC da Organização Mundial da Saúde reestruturou a classificação dos gliomas, de forma que predomina com a inserção de características moleculares além da simples aparência histopatológica.

Face a tais assertivas, importa dizer que é de significativa importância o conhecimento sobre o tema, devido ser uma doença de alta mortalidade e prevalência na população mundial, sendo a segunda neoplasia primária mais comum do sistema nervoso central, somente atrás do meningioma (FATHI; ROELCKE, 2013).

O rápido descobrimento sobre a doença pode influenciar diretamente no prognóstico, tratamento e sobrevida do paciente, afetando demasiadamente a sua qualidade de vida. Ressalta-se sobre o tratamento que desde o primeiro caso de GBM foi relatado, em 1928, ele apresentou significativas melhoras, o tempo médio decorrido entre o diagnóstico e o óbito do paciente, por exemplo, aumentou de 6 para 15 meses entre 1940 e 2010 (BATISTA NETTO; SILVA e NASCIMENTO, 2019).

Face a tais assertivas, importa dizer que este estudo visa informar e alertar o leitor a procurar assistência médica o mais rápido possível a qualquer sinal ou sintoma cerebral incomum, sendo os mais importantes: vômito súbito e constante, cefaleia contínua e persistente, turvação visual, crise epiléptica e rebaixamento do nível de consciência.

Além disso, uma visão geral sobre o assunto, abrangendo: epidemiologia, características clínicas, diagnóstico, prognóstico, tratamento e sobre perspectivas 
futuras. Por fim, sobre a heterogeneidade do glioblastoma e o porquê de ser um tratamento complexo e multimodal (DAVIS, 2016).

\section{OBJETIVOS}

O objetivo desse trabalho foi descrever o glioblastoma, mostrando sua epidemiologia e características histológicas que influenciam no prognóstico e especificações moleculares contribuem na determinação do seu comportamento específico com relação e sua malignidade e agressividade. Objetivou ainda discorrer sobre os tipos de exames diagnósticos e tratamentos disponíveis.

\section{MATERIAIS E MÉTODOS}

Foram incluídas produções cientificas que abordassem o glioblastoma como tema central, escritos em língua estrangeira (inglês) ou língua portuguesa, publicados de 2000 a 2020. Como questão norteadora do estudo elegeu se a seguinte questão: A heterogeneidade do glioblastoma e seus tipos de tratamentos.

A partir do cruzamento dos descritores inicialmente elencados, foram encontrados 107 artigos, no entanto, 14 destes se alinharam aos critérios de inclusão e serviram como fruto da análise. As outras produções encontradas eram somente editoriais e publicações e, portanto, deste modo foram excluídas da pesquisa.

\section{DESENVOLVIMENTO}

O câncer, infelizmente, tem se demonstrado globalmente como uma das principais causa de morte. De acordo com a Organização Mundial da Saúde, aproximadamente entre 6 e 7 milhões têm suas vidas ceifadas em decorrência da referida patologia. As neoplasias malignas são consideradas, as mais importantes causas de mortalidade em nosso meio, sendo responsáveis por mais de $12 \%$ de todas as causas de óbitos no mundo (VIEIRA, 2017).

Dados extraídos do Instituto Nacional de Câncer José Alencar Gomes da Silva (INCA) (2020), traz que o câncer é o nome dado a um conjunto de mais de roo doenças que têm em comum o crescimento desordenado de células, que invadem tecidos e órgãos. No mundo, já se tornou o principal problema de saúde pública, sendo câncer de pulmão o de maior incidência, seguido do câncer de mama. As 
principais causas do aumento de novos casos e mortalidade pela doença no mundo deve-se em parte pelo envelhecimento, crescimento populacional, pela mudança na distribuição e na prevalência dos fatores de risco de câncer, principalmente aos associados ao desenvolvimento socioeconômico.

O câncer é um importante problema de saúde pública em países desenvolvidos e em desenvolvimento e, embora as maiores taxas de incidência de câncer sejam encontradas em países desenvolvidos, dos dez milhões de casos novos anuais de câncer, cinco milhões e meio são diagnosticados nos países em desenvolvimento (PARADA et al., 2008).

\section{I Epidemiologia}

O Instituto Nacional de Câncer (INCA) demonstra que 2\% das neoplasias malignas relatadas no mundo correspondem a câncer do sistema nervoso central (SNC) e no Brasil, até o ano de 2016, havia expectativa de 5.440 casos novos de câncer do SNC em homens e de $4.830 \mathrm{em}$ mulheres (GAILLARD, 2021).

Os tumores do SNC correspondem à segunda causa de morte por câncer pediátrico e a quarta causa de morte por câncer em adultos jovens, sendo dessa forma um problema de saúde de baixa incidência, principalmente na população adulta, porém com alta mortalidade (BRANGER et al., 2008).

O glioblastoma é o tumor glial mais incidente em adultos e idosos e apesar do tratamento, a média de sobrevida é de aproximadamente 14 a 18 meses de vida (citação). Sua incidência aumenta após os 40 anos de idade, com média de 64 anos de idade ao diagnóstico. É mais comum no sexo masculino (I,6:I) e em brancos em relação a negros (2:1) (FRANCO et al., 200o). Por ser um tumor maligno de células astrocitárias, a alta letalidade está relacionada aos seus atributos histológicos de malignidade como anaplasia celular, alto índice proliferativo celular, frequente e extensa necrose, com alta capacidade de infiltração pelas células tumorais.

\subsection{Características clínicas}

Como em todos os tumores cerebrais os sinais e sintomas iniciais do glioblastoma serão inespecíficos ou assintomáticos, podendo ser eles: cefaleia, presente em mais de 50\% dos casos, geralmente de caráter bifrontal e constante, 
irritabilidade, fadiga, apatia, esquecimento, náuseas e vômitos, principalmente, após mudança brusca de posição do corpo ${ }^{12}$. Não costuma cursar com febre na maioria dos casos. Pode ocorrer inicialmente uma sonolência evoluindo até uma síncope, devido ao aumento considerável da pressão intracraniana (PIC) (SOCIETY, 2020).

Por sua rápida expansão e agressividade, em questão de meses os sinais e sintomas vão deixando de ser sistêmicos e se tornando focais, pela questão do efeito de massa e compressão localizada de estruturas encefálicas. Crise epiléptica pode ocorrer entre 18 e $30 \%$ dos pacientes portadores de glioblastoma, que se origina focalmente mas costuma se generalizar secundariamente em questão de segundos (SOCIETY, 2020). Se não reconhecida ou tratada rapidamente pode evoluir para estado de mal epiléptico. Além desses, vamos ter sinais semelhantes ao acidente vascular cerebral como: afasia, perda de controle motor, perda da consciência, hemiparesia e até mesmo plegia. Pelo quadro clínico semelhante, acaba sendo confundido com demência ou distúrbios psiquiátricos. Pode-se ocorrer perda visual por compressão do quiasma ou nervo óptico, levando a papiledema por aumento da PIC (SOCIETY, 2020).

\subsection{Características histopatológicas}

A classificação histológica é de acordo com o tipo citológico predominante do tumor, astrocítico, oligodendrocítico ou misto e, dependendo da presença ou ausência de um determinado número de critérios histológicos de malignidade como a densidade celular, atipia nuclear, mitose, proliferação microvascular ou necrose, pode se determinar o grau de agressividade de I a IV (OLIVEIRA et al., 2009).

Os gliomas de grau I são benignos e sua histologia mais frequente é o astrocitoma pilocítico, de crescimento lento, localizando-se inclusive na fossa cerebral posterior, tronco encefálico, medula espinhal e vias ópticas; os gliomas infiltrantes incluem os de baixo grau (grau II - astrocitoma difuso, xantoastrocitoma pleomórfico) ou malignos como o grau III (astrocitoma anaplásico, oligodendroglioma anaplásico, xantoastrocitoma anaplásico) e o grau IV (glioblastoma, glioma difuso da linha média), que se localizam geralmente nos hemisférios cerebrais e podem evoluir para o glioblastoma grau IV com vascularização anormal e necrose (BELSUZARRI et al., 2018). 
O melhor método de diagnóstico é a ressonância magnética nuclear (RMN) com o uso do contraste gadolíneo. A tomografia computadorizada contrastada pode ser usada na avaliação inicial ou na indisponibilidade da $R M N$ ou se houver contraindicação de seu uso. Contudo o diagnóstico definitivo se obtém através do tipo histológico do tumor através da biópsia, cujos achados histológicos incluem pseudopaliçada, necrose, celularidade aumentada, pleomorfismo, mitoses e proliferação endotelial vascular (OLIVEIRA et al., 2015).

Diversos marcadores moleculares são utilizados para determinar o comportamento específico de um glioblastoma, com relação à sua malignidade, agressividade e resposta terapêutica, tais como os marcadores de superfície CDi33, CDis e CDı7ı. A metilação do gene O6-metilguanina-DNA metiltransferase (MGMT) é notoriamente associada a uma melhor resposta com quimioterapia com agentes alquilantes de DNA (BATISTA NETTO; SILVA e NASCIMENTO, 2019).

Segundo a nova classificação da Organização Mundial da Saúde (OMS) de 2016 dos tumores cerebrais pela primeira vez; os tumores deixaram de ser classificados somente pela histologia, mas também pelos parâmetros moleculares que os compõem (MARQUES; SANTOS, 2018), notadamente as mutações genéticas que afetam as enzimas isocitrato de desidrogenase (IDH) ${ }^{\text {ro }}$. O tipo IDH selvagem se refere à ausência de mutação implicando em pior prognóstico ${ }^{\text {Ir }}$. Em contrapartida, o tipo IDH mutante possui alterações nos genes transcritores, apresentando melhor prognóstico (SIQUEIRA, 2016).

\subsection{Diagnóstico}

Alguns exames de neuroimagem podem ser solicitados para rastreio no caso de suspeita de glioblastoma, podendo ser: tomografia computadorizada, a qual vai se apresentar com margens grossas irregulares iso ou hiperatenuantes, com centro hipodenso, fazendo bastante efeito de massa, com desvio de linha média, apagamento dos giros e sulcos, presença edema vasogênico ao redor. Pode-se ter áreas de hemorragia. Costuma ter intenso realce irregular heterogêneo nas margens (VALLER; TEDESCHI, 2016). 
A ressonância magnética nuclear é o padrão ouro para diagnóstico do glioblastoma. Apresenta algumas características bem-marcadas nas diferentes sequências. Em TI vamos ter uma massa hipo a isointensa na substância branca, com sinal heterogêneo central (VALLER; TEDESCHI, 20ı6). Em T2/FLAIR se apresenta com região hiperintensa circundado por intenso edema vasogênico. $\mathrm{Na}$ sequência SWI os achados mais relevantes são ocasionalmente a presença de calcificações e ausência do sinal da "borda dupla", que serve para diferenciar principalmente dos abscessos cerebrais (VALLER; TEDESCHI, 20I6). Na sequência ADC observa-se componente necrótico cístico sem realce. Mais de 90\% dos glioblastomas apresenta difusão facilitada. A sequência ADC apresenta sensibilidade de 97,6\% e especificidade de 53,1\%. Também existem tipos especiais de ressonância magnética que podem ser úteis, são elas: Angiografia por ressonância magnética e venografia por ressonância magnética, os quais são de grande valor para se observar os vasos sanguíneos cerebrais (PONTES et al., 2012).

Outro exame de neuroimagem que pode ser utilizado é o FDG PET-CT, o qual vai avaliar o aumento do metabolismo de um radiofármaco administrado chamado fluorodesoxiglicose (FDG), geralmente é maior ou semelhante ao da substância cinzenta (PONTES et al., 2012). Ainda pode ser usado a punção lombar do líquido cefalorraquidiano para diagnósticos diferenciais e pesquisa de metástases. Por fim em caso de persistência de dúvida diagnóstica realiza-se a biópsia e o diagnóstico definitivo se dá por meio do anátomo-patológico (PONTES et al., 2012).

\subsection{Tratamento}

De forma geral, o tratamento do glioblastoma, assim como de outros tumores cerebrais, vai ser constituído por ressecção cirúrgica, a principal e mais importante forma de combater a doença, associada a rádio e quimioterapia com agente alquilante de DNA. Devido a sua natureza altamente heterogênea, as opções terapêuticas de combate ao glioblastoma se tornam carentes, devido aos resultados insatisfatórios apresentados por algumas terapias implantadas, apesar de já haver um nítido avanço em relação ao primeiro caso de glioblastoma relatado, em 1928 (FRANCO et al., 2000). 
Por ser um tumor que acomete vários perfis de pacientes e apresenta diversas alterações genéticas como, por exemplo, mutações nos genes das enzimas isocitrato de desidrogenase i e $2\left(\mathrm{IDH}_{\mathrm{I}} / 2\right)$ e a metilação do promotor do gene metilguaninaDNA metiltransferase (MGMT), sendo uma neoplasia constituída por múltiplas células distintas, o que torna o seu tratamento complexo e multimodal ${ }^{3}$. É considerado um tumor de difícil tratamento, possuindo diversos mecanismos de resistência a quimio e radioterapia. No momento, a associação terapêutica ideal é indefinida (FRANCO et al., 200o).

É fundamental o conhecimento quanto ao tratamento cirúrgico, os estudos mostram invariavelmente, uma recorrência do glioblastoma nos pacientes dentro de 6 a 9 meses em média (BATISTA NETTO; SILVA e NASCIMENTO, 2019).

Desde 1970, a associação neurocirurgia mais radioterapia provou dobrar a sobrevida dos pacientes e vai constituir a base do tratamento. Quanto ao tratamento quimioterápico, houve uma grande evolução nos últimos anos. Hoje, os agentes alquilantes como a temozolomida (TMZ), decarbazina e procarbazina surgem como boas apostas (FRANCO et al., 200o). O agente alquilante TMZ foi aprovado em 2005 e apresentou resposta positiva no tratamento (LUCENA et al., 2006). O TMZ é um pró-fármaco que vai ser convertido no plasma em seu metabólico ativo, o monometiltriazenoimidazol carboxamida, o qual vai apresentar biodisponibilidade de 96 a $100 \%$, com boa penetração no sistema nervoso central ${ }^{3}$. Por ser um quimioterápico disponível via oral, vem sendo bastante utilizado, concomitante com a radioterapia, logo após a ressecção cirúrgica. Os pacientes que mais responderam satisfatoriamente a quimioterapia com o $\mathrm{TMZ}$ foram aqueles que apresentam metilação do promotor do gene MGMT ((BATISTA NETTO; SILVA e NASCIMENTO, 2019).

Diante desse cenário reservado e incerto, algumas novas medidas terapêuticas estão sendo estudadas e testadas e vão constituir as perspectivas futuras no tratamento do glioblastoma, como: As terapias-alvo, imunoterapias e fitoterapias (BATISTA NETTO; SILVA e NASCIMENTO, 2019).

Desde o primeiro caso de glioblastoma descrito na literatura até o prezado momento, ainda não falamos em cura quando nos referimos ao tratamento do glioblastoma, mas sim, há uma maior sobrevida e melhor qualidade de vida. Em 
pacientes que optam por não se tratar, falamos em uma sobrevida de 4 a 6 meses em média. Já em pacientes que se submetem a uma terapia multimodal, essa sobrevida aumenta para I4 meses (BATISTA NETTO; SILVA e NASCIMENTO, 2019).

\section{CONCLUSÃO}

De acordo com o estudo realizado, podemos concluir que o glioblastoma é um dos tumores cerebrais primários mais prevalente em adultos na população mundial e, muitas vezes, por ser assintomático no início ou por ter sintomas inespecíficos acaba sendo uma doença de diagnóstico difícil e tardio e assim apresenta um rápido crescimento com sintomas agressivos.

Dessa forma, devido sua heterogeneidade genética o glioblastoma tem um tratamento complexo, constituído basicamente por ressecção cirúrgica mais radioterapia na maior parte das vezes e concomitantemente pode-se realizar quimioterapia com agentes alquilantes de DNA. No entanto, o prognóstico da doença é ruim, apresenta uma sobrevida de 4 a 6 meses em pacientes que optam pelo não tratamento e 14 meses em média em pacientes com tratamento multimodal.

Fica como sugestão para futuros estudos um estudo clínico com paciente acometido pelo referido tumor. A limitação deste estudo pautou-se em encontrar artigos dos últimos cinco (5) anos.

\section{REFERÊNCIAS}

AARON, C. T. et al. Tratamento do gliobastoma: estado da arte e direções futuras. Cancer Journal for Clinicians, v.70, n.4, p.231-314, 2020. Disponível em: https://acsjournals.onlinelibrary.wiley.com/doi/full/ı.3322/caac.21613 Acesso em: ıo out.2021.

BATISTA NETTO, Jairo; SILVA, Thamyres Figueredo; NASCIMENTO, Gabriel Vinicius Ferreira do. Glioblastoma: patogênese e tratamento. Revisão da literatura. J Bras Neurocirur, v.30, n.3, p.233-243, 2019. Disponível em: https://www.abnc.org.br/jbnc_art_down.php?arquivo=I352 Acesso em: Io out. 202I.

BELSUZARRI, et al. Heteregeneidade dos tumores cerebrais. Arquivos Brasileiros de Neurocirurgia, v.3, n.8, 2018. Disponível em: 
https://www.researchgate.net/publication/323755449_Heterogeneidade_dos_tumores _cerebrais Acesso em: io out.2021.

BRANGER-FIGARELLA, D. et al. Classification histologique et moléculaire des gliomesHistological and molecular classification of gliomas. ScienceDirect, v.I64, n.6-7, p.505-515, 2008. Disponível em: https://www.sciencedirect.com/science/article/abs/pii/Soo3537870800297X?via\%3 Dihub\#: :text=Classification\%2ohistologique\%2oet,classification\%20of\%2ogliomas Acesso em: Io out.2021.

CAMBRUZI, Eduardo. O papel das mutações $I D H_{I} / 2$ na patogênese dos glioblastomas secundários. Jornal Brasileiro de Patologia e Medicina Laboratorial, v.53, n.5, 2017. Disponível em: https://www.scielo.br/j/jbpml/a/NfxfdTNZ4qzf9 ${ }_{9} \mathrm{Qhsg}_{9} \mathrm{C}_{9} \mathrm{HFt} / \mathrm{abstract} /$ ?lang=pt\# Acesso em: ro out.2021.

FRANCO, Clélia Maria Ribeiro et al. Gliomas múltiplos: casos ilustrativos de quatro formas de apresentação. Arq Neuropsiquiatr., v.58, n.I, p.150-158, 2000. Disponível https://www.scielo.br/j/anp/a/9WPmzFKDJwChxTwwR97TPgJ/?lang=pt\# Acesso em: Io out. 2021.

GAILLARD, Frank. Gliobastoma. 2021. Disponível em: https://radiopaedia.org/articles/4910 Acesso em: Io out. 202I.

INCA - Instituto Nacional de Câncer José Alencar Gomes da Silva. Estimativa 2or6: incidência de câncer no Brasil. Rio de Janeiro: Ministério da Saúde/INCA; 2015.

INCA - Instituto Nacional de Câncer José Alencar Gomes da Silva. Estimativa 2014: incidência de câncer no Brasil. Rio de Janeiro: Ministério da Saúde/INCA, 2014. LUCENA, Rita de Cássia G. et al. Correlação clínico-topográfica em glioblastomas multiformes nas síndromes motoras: significados fisiopatológicos. Arq Neuropsiquiatr., v.64, n.2-B, 44I-444, 2006. Disponível em:

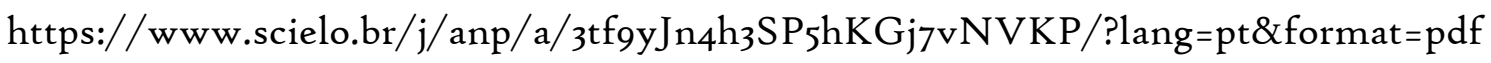
Acesso em: Io out. 2021. 
MARQUES, Daniel Fernandes; SANTOS, Marcos. Gliomas de alto grau. Cap.2, 2018.

OLIVEIRA, Viviane Daneluci de et al. Glioblastoma: base conceitual e perspectivas futuras de tratamento. Revista Uniara, v.12, n.2, dez.2009. Disponível em: https://www.uniara.com.br/legado/revistauniara/pdf/23/denilson_16.pdf Acesso em: Io out. 202I.

PARADA, Roberto Parada.; ASSIS, Mônica de.; SILVA, Ronaldo Corrêa Ferreira da.; ABREU, Maria Fátima.; SILVA, Marcos André Felix da.; DIAS, Maria Beatriz Kneipp.; TOMAZELLI, Jeane Glaucia. A política nacional de atenção oncológica e o papel da atenção básica na prevenção e controle do câncer. Rev. APS, v. II, n. 2, p. 199-206, abr./jun. 2008. Disponível em http://www.aps.ufjf.br/index.php/aps/article/viewFile/263/Ioo.>. Acesso em: is out.202I.

PONTES, Luciola de Barros et al. Glioblastoma: enfoque no tratamento de pacientes idosos. Einstein. v.Io, n.4, p.512-518, 2012. Disponível em: https://www.scielo.br/j/eins/a/bfd ${ }_{3} \mathrm{Qg}_{4} \mathrm{G} 8 \mathrm{mDqn} 8 \mathrm{XFyw} V \mathrm{rWqg} / \mathrm{abstract} /$ ?lang=p t\# Acesso em: Io out. 2021.

SIQUEIRA, M.G. Tratado de neurocirurgia. r.ed. São Paulo: Manole, 2016.

SOCIETY. Testes for Brain and Spinal Cord Tumors in Adultos. 2020. Disponível em: https://www.cancer.org/cancer/brain-spinal-cord-tumors-adults/detectiondiagnosis-staging/how-

diagnosed.html\#: :text=DIAGNOSIS\%2C\%20AND\%20STAGING-

,Tests\%20for\%2oBrain\%20and\%20Spinal\%20Cord\%20Tumors\%20in\%2oAdults,-

Brain\%20and\%20spinal Acesso em: ro out.2021.

VAlLER, L.; MIN, L.L.; TEDESCHI, H. Manual de Neuro-Oncologia da UNICAMP. 2oi6. Disponível em: https://issuu.com/adciencia/docs/manual_de_neuro-oncologia_da_unicam Acesso em: Io out.2021. 
VIEIRA, Sabas Carlos. Câncer. Consenso da Sociedade Brasileira de Mastologia.

Teresina: EDUFPI, 2017. Disponível em: <

https://www.sbmastologia.com.br/medicos/wp-

content/uploads/2018/o3/C\%C3\%A2ncer-de-Mama-Consenso-da-SBM-Regional-

Piau\%C3\%AD-2017.pdf>. Acesso em: I5 out.2021. 